\title{
D2D-based QoS prediction analysis in beyond 5G $\mathrm{V} 2 \mathrm{X}$
}

\author{
Tomasz Mach \\ Samsung R\&D Institute \\ Staines, UK \\ tomasz.mach@samsung.com
}

\author{
Galini Tsoukaneri \\ Samsung R\&D Institute \\ Staines, UK \\ g.tsoukaneri@,samsung.com
}

\author{
Daniel Warren \\ Samsung R\&D Institute \\ Staines, UK \\ dan.warren@samsung.com
}

\begin{abstract}
With mobile networks expected to support services with stringent reliability, availability, latency and throughput metrics, resulting in a more complex concept of Quality of Service (QoS), the ability to predict QoS variation and adapt the flow of traffic accordingly has become a critical requirement for some use cases. For example Connected and Automated Mobility applications could use QoS prediction to reduce the speed of an autonomous vehicle if network performance is going to deteriorate, and critical information needs to be conveyed. Current state-of-the-art approaches on QoS prediction are mostly focused on core network $(\mathrm{CN})$, which is complex and suboptimal in some scenarios. In this paper, we introduce the concept of UE-based QoS prediction, discuss its motivation, and propose novel lightweight device-to-device (D2D)-based coverage prediction framework in RAN, based on a generalized D2D use case, applicable to multiple industries. We discuss how the proposed mechanism may be complementary to the $\mathrm{CN}$-based prediction, analyse its performance and provide simulation results of the proposed framework to showcase its advantages. Finally, we study how the D2D prediction information could be used to trigger 5G RAN protocol adaptations, such as predictive data scheduling in MAC.
\end{abstract} MAC

Keywords - V2X, Predictive QoS, D2D, B5G, RAN, RRC,

\section{INTRODUCTION}

Mobile networks are increasingly expected to support the reliable delivery of messages for mission critical services. However, networks still experience variations in connection quality, so ensured reliable delivery of messages can be impacted by changes in connection throughput.

In an effort to counteract this, QoS prediction mechanisms in $5 \mathrm{G}$ systems based on in-advance notifications have recently been studied among different industry groups, including 3GPP [5, 6], ETSI [7], GSMA [8] and 5GAA [2], as well as in research projects such as '5GCroCo' [1]. Without QoS prediction, an application can only adapt its behavior reactively, after a QoS change has occurred. By using prediction information, applications could proactively adapt, especially in mission-critical use cases. While the idea of predictive QoS has been initiated by telecom and automotive industry cooperation [2], it is expected that other verticals such as Industry 4.0 could also benefit.

Preliminary efforts are mostly based on Core Network $(\mathrm{CN})$ based solutions, with more work expected to better understand QoS prediction use cases, and develop corresponding requirements. This paper focuses on a novel D2D UE-based coverage prediction in RAN, to provide additional measurements, which may be used complementary to CN-based QoS prediction.

In this paper, we look into the $\mathrm{CN}$-based predictive QoS frameworks and discuss their shortcomings. We then present a novel, lightweight D2D-based QoS prediction framework that allows for autonomous UE prediction. We discuss how it could be implemented in automotive applications and how inadvance knowledge of degrading radio conditions could be leveraged in 5G MAC protocol. We also propose an enhanced to the state-of-the-art approach that updates the inter-message gap based on the signal degradation ratio experienced by the vehicles and perform a feasibility study to evaluate the performance of the proposed framework.

\section{ON THE NEED FOR UE AUTONOMOUS QOS PREDICTION}

Applications that are dependent on the support of extreme network performance attributes (high bandwidth, low latency, high reliability), are by implication, likely to be adversely affected when those attributes are not available. Where such applications and services are part of Business-to-Business (B2B) or Business-to-Business-to-Customer (B2B2C) contracts, it is also likely that unpredicted service quality issues will result in the invocation of commercial penalty clauses, and where mission-critical aspects are included, the potential for Mobile Network Operators to have liability for some or all of commercial losses incurred as a result.

Predictive QoS enables the network to deliver information about QoS changes that are likely to occur, through InAdvance QoS Notification (IQN) messages from the IQN producer and the Prediction Function (PF) in the $\mathrm{CN}$. This allows the UE to adapt its behavior to request any critical information ahead of any degradation in QoS that it has been informed is likely to occur, and also to prepare applications for the impending change in QoS characteristics. This collection of information and adjustment in behavior is expected to make some key $\mathrm{B} 2 \mathrm{~B}$ or $\mathrm{B} 2 \mathrm{~B} 2 \mathrm{C}$ applications viable, which would otherwise have not been able to function if reactive behavior to QoS change was employed.

The PF collects connection data and generates predictions through specific algorithms based on collected KPI measurements. An architecture for predictive QoS based on interaction between the RAN and $\mathrm{CN}$ may be efficient in many situations, but can suffer in certain scenarios from

a) High cost and complexity - end to end predictive QoS feature needs to be implemented both in the UE and the network - in practice, e.g. for a vehicle UE it may be difficult to ensure when roaming in the 
network which does not support such feature in some parts of the network. Also theoretically. some $5 \mathrm{G}$ networks (5GC) may still use EUTRAN (LTE) rather than NR in RAN which may limit end to end QoS prediction capability, however it remains to be seen how likely this configuration would be in practice. Furthermore, some MNOs may not support sharing QoS prediction data between their networks in the future so sharing inter-frequency or inter-RAT cell coverage prediction measurements directly between vehicle UEs may be beneficial.

b) Decreased reliability in poor coverage and no short-range communication support - predictive QoS feature assumes suitable connectivity in RAN i.e. availability of radio resources allowing radio bearers establishment on $\mathrm{Uu}$ interface to send prediction data, however due to the dynamic nature of wireless communication in some cases this is not possible i.e. poor or out of coverage scenarios which are often experienced due to physical obstacles or in rural road areas. Also RAN congestion could impact the interference limited network coverage. This may increase delay in sending the prediction information in RAN. This is shown in Fig. 1 where the UE based coverage prediction on sidelink / PC5 interface could be used when prediction on Uu interface is not possible due to the cell edge or coverage hole radio conditions. Some of these approaches were recently explored by 5GAA [10]. In addition, leveraging synergy between integrated availability of PC5 and $\mathrm{Uu}$ interfaces in Cellular Vehicle-to-Everything (C$\mathrm{V} 2 \mathrm{X}$ ) for better applications support may give the competitive advantage to $\mathrm{C}-\mathrm{V} 2 \mathrm{X}$ in comparison to competing V2X technologies such as DSRC, ITSG5 or hybrid approaches. It should be also highlighted, that as the NW role is reduced in the coverage prediction using sidelink / PC5 interface, e.g. for a vehicle UE in case of potential road incident, the MNO liability may be reduced.

c) Centralized architecture and limited QoS change granularity - in case predictive QoS feature is only $\mathrm{CN}$-based, it is assumed sufficient supporting KPI measurement data is available for the QoS prediction in the network, however in some cases e.g. collected radio coverage statistics at specific UE position may only provide averaged information about the

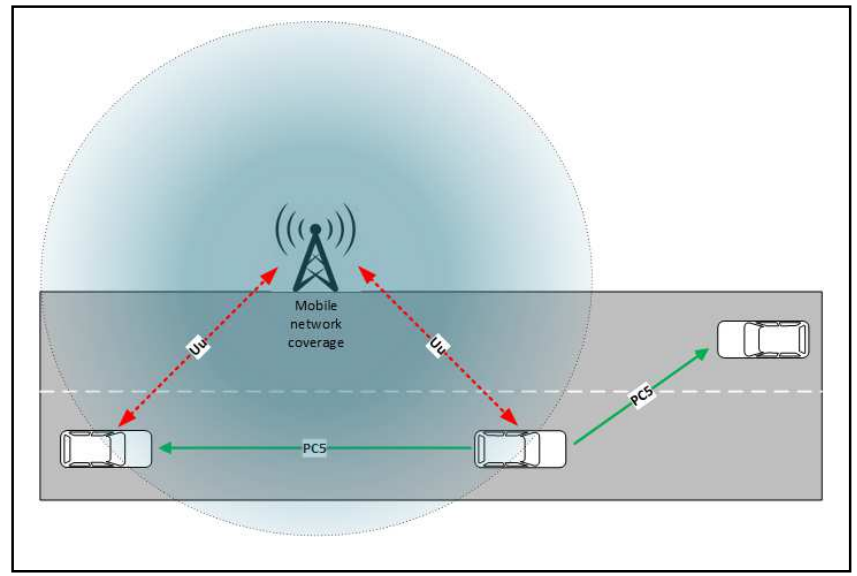

Fig. 1. UE based prediction on PC5 interface in and out of coverage coverage eliminating fast coverage changes due to the dynamic radio resource usage and corresponding real time coverage change information - such limitation is especially relevant to the interference limited coverage e.g. LTE RSRQ measurements. Furthermore, predicting QoS in a distributed manner is better aligned with adhoc $\mathrm{V} 2 \mathrm{~V}$ communication characteristics.

Alternatives to $\mathrm{CN}$-based QoS prediction could provide more dynamic QoS predictions, are less complex and may potentially integrate with limited prediction capability e.g. coverage only. Such mechanisms can either be UE or RAN based, and could exploit the synergy between available shortrange (sidelink/PC5 interface) and long-range communication (Uu interface) (Fig. 2). Distributed UE autonomous scheduling to transmit V2V messages is still possible out of coverage in 5G sidelink mode 2 [3] (mode 4 in LTE) and thus has no RAN coverage dependency. These solutions can be deployed in isolation, or as part of CN-based techniques for further improvements in predictions.

\section{D2D Coverage Change PREDiCTION}

To predict mobile coverage changes, we propose a framework based on received network signal measurements that are periodically shared via D2D connections with surrounding UEs. Cell measurements are combined with the source UE's location and measured cell identification information to allow cell-specific signal level tracking in the receiving UEs (Fig. 3).

The combination of network signal measurements from vehicle B with its operational information (location, speed, and heading) enables mobile coverage change prediction, and may trigger corresponding safety actions, such as SAE [10] driving automation level reconfiguration in vehicles $\mathrm{A}$ and $\mathrm{C}$. This scheme allows QoS prediction to be passed from devices with network coverage to those without, and vice versa. It can also provide significant advantages in areas with poor network coverage, or areas where the network coverage

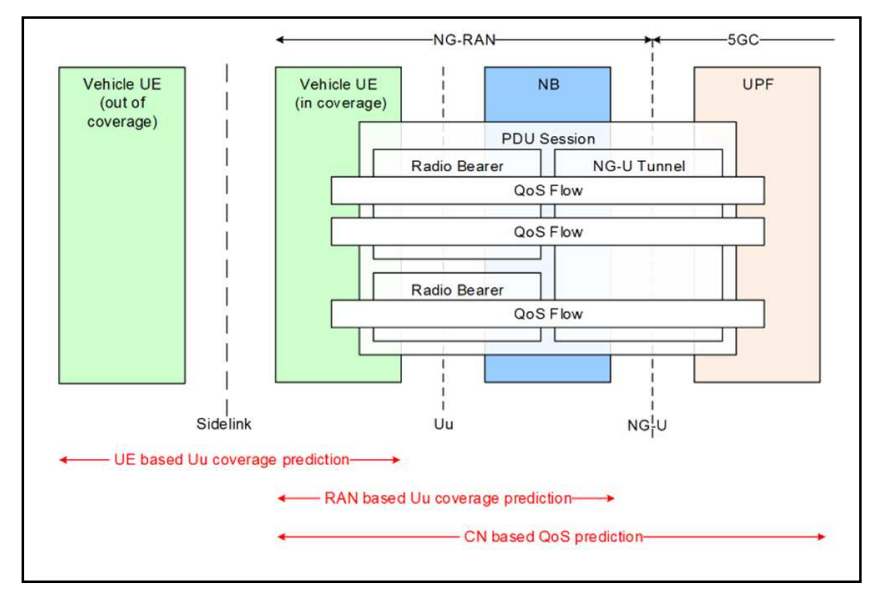

Fig. 2. UE/RAN coverage prediction complementary to $5 \mathrm{G} \mathrm{CN}$ prediction. 


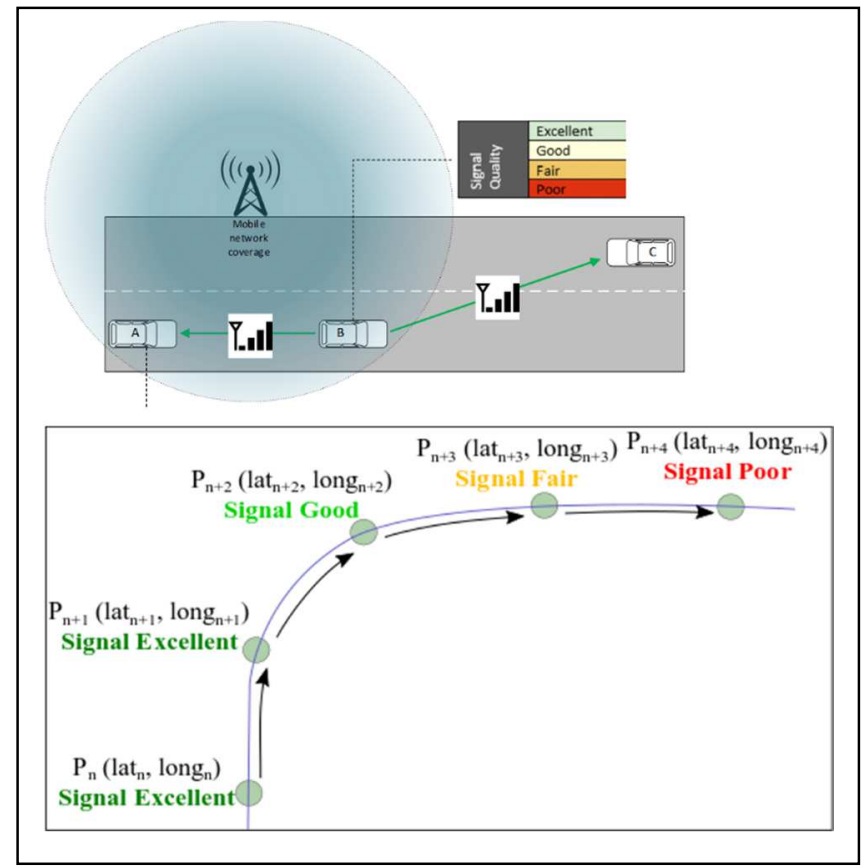

Fig. 3. Coverage change prediction based on V2X scenario.

significantly changes within a short time (e.g. a deep tunnel on a motorway).

\section{Coverage Change Prediction Signaling}

In $\mathrm{C}-\mathrm{V} 2 \mathrm{X}$ communications, in parallel to Uu interface, there is a sidelink (PC5) interface defined for direct vehicle UE to UE communication (Fig. 1). To allow the prediction of mobile network coverage change in a vehicle UE, it is proposed to extend the 3GPP Radio Resource Control (RRC) protocol cell measurement reporting mechanism to PC5 interface used in $\mathrm{C}-\mathrm{V} 2 \mathrm{X}$ direct communications between vehicles (currently this mechanism is only used on $\mathrm{Uu}$ interface). As a result, existing measurements events (standardized for the measured serving cell or neighbor cells quantities) may be used to preconfigure, trigger and report observed mobile radio signal coverage changes by a vehicle to the locally surrounding vehicles. Proposed coverage change prediction mechanism relies on the existing cell measurements available in the UE and their performance and it is not expected that any new requirements related to the UE measurements capability or accuracy would be required.

Fig. 4 shows and example of the 'serving cell lost' scenario prediction based on the existing RRC Event A2 ('Serving cell becomes worse than threshold') sent in V2X messages either via PC5 or Uu interface. It could be noted, that in the receiving vehicle travelling in the opposite direction to the source vehicle, the measured radio signal quantity change is inversed and as a result event A2 is interpreted as event A1 'Serving cell becomes better than threshold'. If the threshold is predefined as a minimum received signal level (see LTE RSRP Q-RxlevMin and RSRQ Q-QualMin defined in [11]), triggered event $\mathrm{A} 2$ could be interpreted (by the receiving vehicle UE) as the sending vehicle UE moving out of the network coverage and triggered event $\mathrm{A} 1$ as the sending vehicle UE entering the network coverage.

It is envisioned that two coverage change prediction signaling options could be possible:

- Basic coverage change prediction - Measurement event triggering in the vehicle UE could be

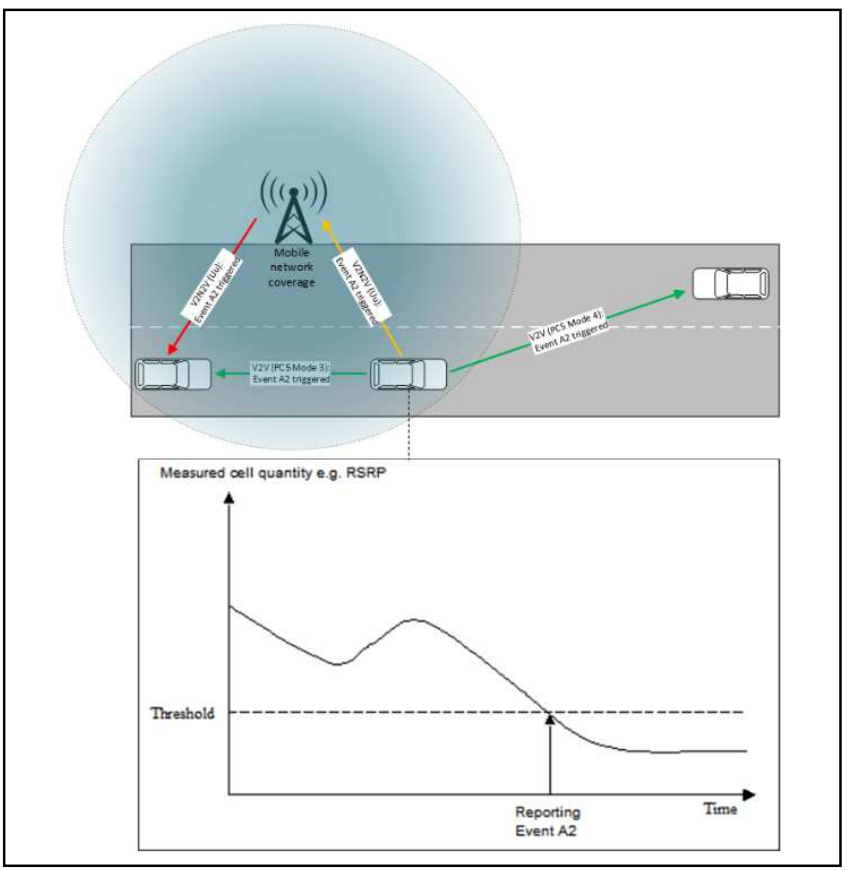

Fig. 4. Coverage change prediction using RRC Measurement Event A2.

predefined statically e.g. a binary in or out mobile network coverage flag with related cell frequency and cell identifier could be periodically broadcasted in V2X messages such as defined in Cooperative Awareness Message (CAM) [12] or Basic Safety Message (BSM) [13] standards. Other types of V2V messages could be also used such as Collective Perception Message recently defined in ETSI ITS standard [15]. This message shares the vehicle sensor information with neighboring cars and including QoS prediction information from the V2X modem, could be a first step to expand V2V Collective Perception Service to a new dimension of the radio communications. This approach does not require request / response signaling. Such approach may be easier to implement and could be beneficial also for the hybrid V2X communication using DSRC or ITS-G5 in parallel with cellular Uu interface.

- Advanced coverage change prediction Measurement events triggering in the vehicle UE could be configured dynamically e.g. by other vehicles, network, or Road Side Units (RSUs) etc. (Measurement Request). Triggered measurement event information (flag) could be transmitted to the surrounding vehicles in $\mathrm{C}-\mathrm{V} 2 \mathrm{X}$ radio access protocol stack. To this end, RRC layer messages may be extended to PC5 interface e.g. similar message to the existing RRC Measurement Report message may be defined on the sidelink.

Because RRC protocol currently does not support operational vehicle information such as location, speed or heading, the information about a triggered radio coverage event would need to be combined with such information available in higher application layers e.g. in V2X CAM or BSM messages of the vehicle UE protocol stack implementation.

In the receiving $\mathrm{V} 2 \mathrm{X}$ system, combining triggered measurement events information with a periodic reception of 
the vehicle location and moving direction extracted from received V2V CAM or BSM Messages (Fig. 3), enables continuous tracking and prediction of the network coverage change for a vehicle UE following other vehicle. Similar reporting approach is already used in BSM messages standard [13] e.g. for a Control Loss Warning when a vehicle control loss event flag is enabled and broadcasted to surrounding vehicles after activation of Antilock Brake System, Traction Control Loss or Stability Control Loss.

It is expected that combining the measured cell level and quality with operational vehicle information i.e. location, speed, heading, would be enough to distinguish different vehicle UEs and track them as independent sources of coverage change information. Information from more than one source about the same cell loosing coverage would be useful as it could increase the reliability in the coverage prediction information algorithm.

With this distributed approach, C-V2X modem in a vehicle could be considered another sensor, sharing its control plane data (serving and neighbor cells RRC measurement events) with surrounding vehicles via PC5 to support cooperative observation services and increase environment awareness important for improved road safety.

\section{Predictive Data Scheduling IN MAC}

Building upon the D2D-based solution described above, network measurements can be used to enhance the QoS predictions. One of the functions of 5G MAC protocol [4] is data scheduling. For instance in uplink, scheduling is typically based on the UE buffer status reported to the $5 \mathrm{G}$ base station (gNB), that allows the gNB to schedule the radio resources to the UEs along with the radio conditions reported by the UE. By predicting the radio conditions based on the D2D information sharing from the preceding V2X UEs (Fig. 5), the scheduler expects a proactive increase in the UE's request for data transmission and allows more radio resources to be granted. As a result, by implementing the predictive data scheduler, the overall performance of the system is improved by increasing the effective throughput (goodput) in the cell.

\section{D2D Coverage PREDiction Feasibility StUdy}

To assess the feasibility and performance of the proposed D2D prediction framework, we have performed simulations using an updated version of the ns-3 simulator developed in [14]. Our simulation parameters can be found in Table I. Please note that each message with prediction information is

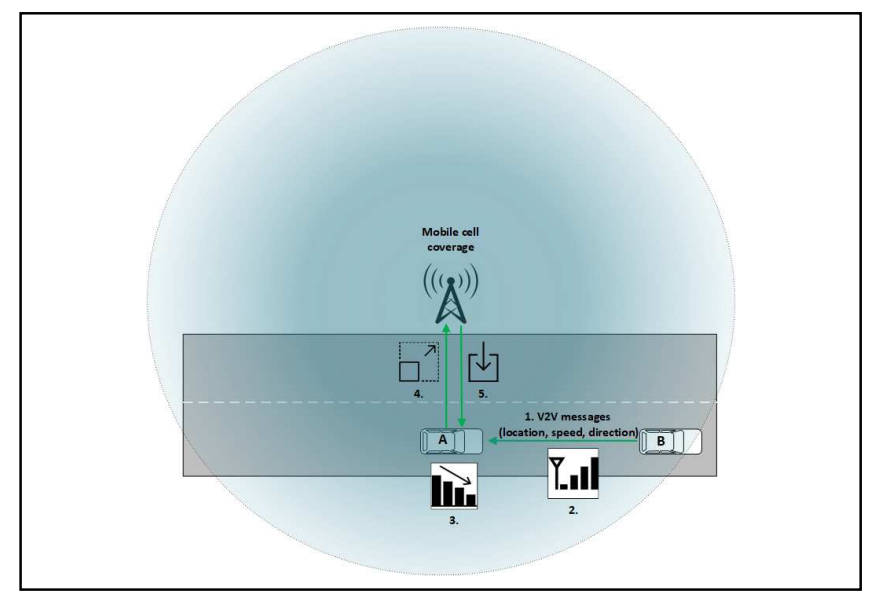

Fig. 5. Predictive MAC scheduling based on in-advance QoS change detection. received 3 times (before it can be acted upon) to enhance the reliability of the prediction mechanism.

For our simulations, we assumed a motorway scenario where the road consists of 3 lanes in each direction. Vehicles are placed in each lane with weighted probabilities of 0.45 , 0.3 and 0.25 for lanes 1 to 3 respectively (we assume that lane 1 is the slowest lane, while lane 3 is the fastest one). Vehicle speed is kept constant throughout the experiment at 80, 95 and $120 \mathrm{~km} / \mathrm{h}$ for lanes 1 to 3 respectively. We also evaluated a scenario in a city center where the road consists of 2 lanes and vehicles move with a speed of $30 \mathrm{~km} / \mathrm{h}$. We assume that vehicles may communicate with the base station to exchange vehicle-specific information (not V2V), while they use the sidelink to communicate with each other to exchange CAM messages [12]. The QoS predictions are based solely on the CAM messages received in the sidelink, thus accounting for the scenarios where the base station signal is weak or nonexistent, and allowing the vehicles to take action before the signal becomes unusable.

In our simulations, we consider two separate cases for the CAM transmission. In the first case, we follow the ETSI ITS standard recommendation and update the transmission periodicity of the CAM messages based on the speed of the vehicles [12]. In the second case, we follow a novel approach and change the transmission periodicity of the CAM messages based on the Signal Degradation Ratio (SDR) given by the RSRP. This method extends the state of the art approaches and can be used either instead or in addition to the speed-based periodicity [12]. This allows a UE to inform its neighboring vehicles about sudden changes in the signal strength more proactively, giving them enough time to adjust their behavior to the new signal conditions.

Fig. 6 shows how the inter-message gap is updated when the CAM transmission periodicity is based on the SDR for different speeds. We can see that the inter-message gap is adapted based on the SDR so that UEs can more quickly and accurately inform their neighbors about changes in the signal level. Please note that the initial inter-message gap for the four speeds is different, as proposed by $3 \mathrm{GPP}$. Since $3 \mathrm{GPP}$ does not specify exact values for the inter-message gap and different speeds, we randomly selected 100, 90, 80 and $70 \mathrm{~ms}$

TABLE I.

\begin{tabular}{|l|c|}
\hline \multicolumn{1}{|c|}{ Parameter } & Value Used \\
\hline Cell radius & $5 \mathrm{~km}$ \\
\hline Number of vehicles & $2-50$ \\
\hline Length of CAM message & Up to 300 bytes \\
\hline UE Tx power & $23 \mathrm{dBm}$ \\
\hline UE speed & $30-120 \mathrm{~km} / \mathrm{h}$ \\
\hline $\begin{array}{l}\text { CAM repetition for coverage } \\
\text { prediction reliability }\end{array}$ & 3 \\
\hline
\end{tabular}

for $30,80,95$ and $120 \mathrm{~km} / \mathrm{h}$. Although the actual intermessage gaps used in real scenarios might be different to the ones used in this experiment, the overall trend remains the same. 
Another important aspect of V2X applications is the requirement that CAM messages are received at the destination in advance, giving the destination enough time to make a decision and take an action (e.g. break the vehicle, change lane). Currently, it has been proposed that the QoS Prediction Notification (QPN) time, i.e. the time period between detecting the upcoming coverage change and experiencing it, is $2.3 \mathrm{~s}$ to allow for the recommended $2 \mathrm{~s}$ intervehicle road safety gap and the reception of three $\mathrm{V} 2 \mathrm{~V}$ messages indicating the coverage change, each requiring $0.1 \mathrm{~s}$. Based on that, CAM messages need to be received at the destination at least 2.3 seconds before the action needs to be taken. Therefore, we also evaluate the number of messages that met the $2.3 \mathrm{~s}$ deadline in terms of the distance between the vehicles and the number of vehicles using the sidelink (Fig. $7)$. In this experiment, we followed the configuration and methodology of [14], and we assumed that the coverage prediction information was successfully received if all three CAM message repetitions were received. We can see that for longer inter-vehicle distances the percentage of CAM messages reaching their destination in time falls significantly, due to the decreased sidelink transmission performance getting closer to its maximum range.

\section{CONCLUSION}

In this paper, $\mathrm{CN}$-based predictive QoS was analyzed and its main shortcomings were identified. A new agile D2Dbased QoS prediction framework was proposed using autonomous UE prediction when lightweight and fast prediction is required. We demonstrated how it could be implemented in automotive applications (however we believe it may also benefit other verticals) and also how in-advance knowledge of degrading radio conditions could be leveraged in 5G MAC protocol. We also proposed an enhanced to the state-of-the-art approach that updates the inter-message gap based on the signal degradation ratio experienced by the vehicles. We then performed a feasibility study using the ns3 simulator to evaluate the performance of the proposed framework.

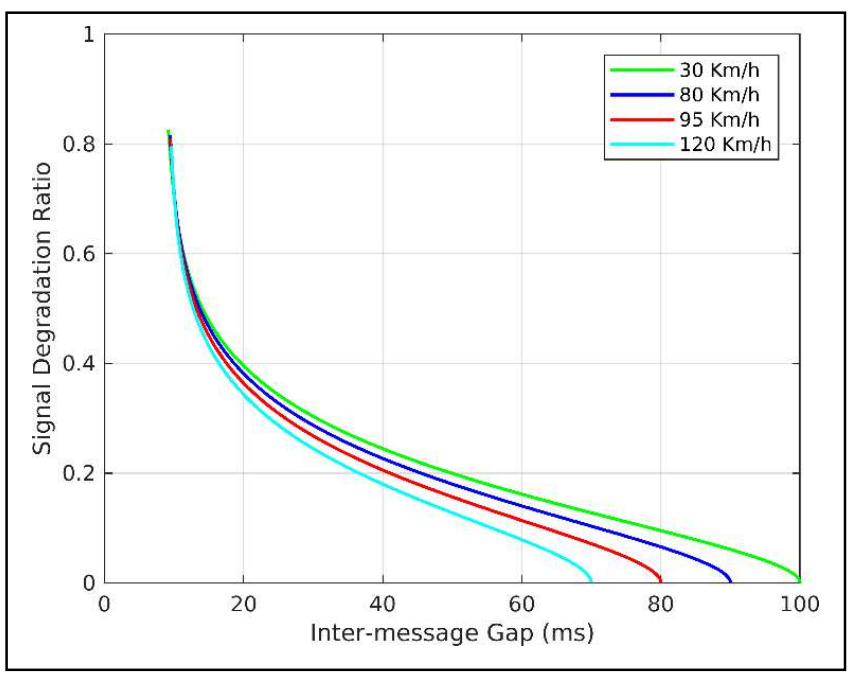

Fig. 6. Inter-message gap update based on SDR for different UE speeds.

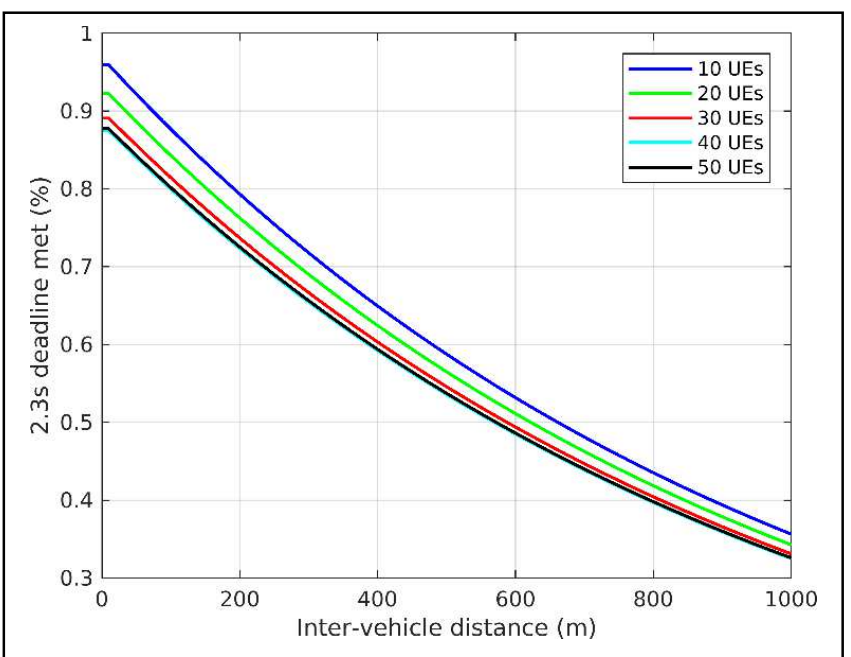

Fig. 7. $\%$ of messages meeting $2.3 \mathrm{~s}$ deadline for different \# of vehicles and distances.

\section{ACKNOWLEDGMENT}

This research work was sponsored in part by the European Union's Horizon 2020 research and innovation programme under Grant No. 871249 (LOCUS).

\section{REFERENCES}

[1] 5GCroCo (2019-09-10) 'Deliverable D1.2, 5GCroCo First Intermediate Report'

[2] 5G Automotive Association whitepaper 'Making 5G Proactive and Predictive for the Automotive Industry' 2020

[3] 3GPP TS 38.300 V15.9.0 (2020-04) 'NR; Overall description; Stage-2'

[4] 3GPP TS 38.321 V15.8.0 (2020-01) 'NR; Medium Access Control (MAC) protocol specification'

[5] 3GPP TS 22.185, V14.4.0 (2018-06): Service Requirements for V2X Services, Stage 1 (R14)

[6] 3GPP TS 22.186, V16.2.0 (2019-06): Enhancement of 3GPP Support for V2X Scenarios

[7] ETSI GR MEC 022 V2.1.1 (2018-09): Study on MEC support for V2X Use Cases

[8] GSMA NG.116 V1.0 (2019-05): Generic Network Slice Template version 1.0

[9] Society of Automotive Engineers (SAE), "Levels of Driving Automation (SAE J3016)", https://www.sae.org/news/2019/01/saeupdates-j3016-automated-driving-graphic

[10] 5G Automotive Association whitepaper '5GS Enhancements for Providing Predictive QoS in C-V2X' 2020

[11] 3GPP TS 36.331 V14.8.0 (2018-09) "Evolved Universal Terrestrial Radio Access (E-UTRA); Radio Resource Control (RRC); Protocol specification"

[12] ETSI EN 302 637-2 V1.3.2 (2014-11): "Intelligent Transport Systems (ITS); Vehicular Communications; Basic Set of Applications; Part 2: Specification of Cooperative Awareness Basic Service"

[13] SAE J2735 "V2X Communications Message Set Dictionary" 2020

[14] F. Eckermann, M. Kahlert, and C. Wietfeld. Performance analysis of c$\mathrm{v} 2 \mathrm{x}$ mode 4 communication introducing an open-source $\mathrm{cv} 2 \mathrm{x}$ simulator. arXiv preprint arXiv:1907.09977, 2019

[15] ETSI TR 103562 V2.1.1 (2019-12): Analysis of the Collective Perception Service; Release 2 